\title{
Correction to: The Skin and Diabetes
}

J. W. J. Lasschuit, J. R. Snaith, and J. W. Frew

\section{Correction to: C. Salavastru et al. (eds.), Skin and the Heart, https://doi.org/10.1007/978-3-030-54779-0_18}

This book was inadvertently published with incorrect captions to the figures 2 and 3 in chapter 18 . This has now been corrected in the book. 